\title{
Medición objetiva del comportamiento sedentario y la actividad física, y de sus efectos en la variabilidad de la frecuencia cardiaca, en adultos jóvenes que residen en una altitud moderada
}

\author{
Objective measurement of sedentary behavior and physical activity, and their effects on \\ heart rate variability, in young adults that live in a moderate altitude
}

\author{
Jaime T. Rojas ${ }^{1 *}$ y Carlos A. Suárez ${ }^{1,2}$
}

${ }^{1}$ Facultad de Ciencias de la Salud, Universidad de Ciencias Aplicadas y Ambientales, ${ }^{2}$ Escuela de Ciencias de la Salud y el Deporte, Universidad Sergio Arboleda. Bogotá, Colombia

\section{Resumen}

Antecedentes: El ejercicio físico y el entrenamiento de resistencia aeróbica se han asociado con un aumento de la variabilidad de la frecuencia cardiaca, mientras que el tiempo sedentario se ha asociado con una disminución de la variabilidad de la frecuencia cardiaca. Objetivo: Determinar si la variabilidad de la frecuencia cardiaca medida durante el reposo se relaciona con el tiempo sedentario y el tiempo utilizado en diferentes intensidades de actividad física, determinadas a través de un método objetivo (acelerometría), en un grupo de adultos sanos que residen a una altura de 2600 metros sobre el nivel del mar (m.s.n.m.). Método: Se realizaron mediciones de acelerometría a 99 individuos durante una semana y una medición de variabilidad de la frecuencia cardiaca en reposo. De acuerdo con la acelerometría, se realizó una división en tiempo sedentario y tiempo de actividad física de intensidad ligera, moderada y vigorosa. Para analizar la variabilidad de la frecuencia cardiaca se utilizaron parámetros en el dominio del tiempo (intervalos normales [NN], desviación estándar de los intervalos normales [SDNN], raíz cuadrada del promedio de las diferencias al cuadrado entre intervalos normales adyacentes [RMSSD]) y de la frecuencia (potencia de la baja frecuencia [LF], potencia de la alta frecuencia [HF], LF/HF) para analizar la variabilidad de la frecuencia cardiaca. Mediante de modelos de regresión se buscó la asociación entre las variables de actividad física y de variabilidad de la frecuencia cardiaca. Resultados: Se presentó una asociación negativa entre los intervalos NN, la SDNN y el tiempo sedentario, así como asociaciones positivas entre el intervalo NN y la actividad física ligera, moderada y vigorosa, al igual que entre la actividad física vigorosa y las potencias de LF y HF. Todas las asociaciones anteriores fueron significativas ( $p<0.05)$. Conclusiones: En adultos jóvenes que residen a 2600 m.s.n.m., el tiempo sedentario reduce la variabilidad de la frecuencia cardiaca, mientras que la actividad física vigorosa aumenta dicha variabilidad.

Palabras clave: Variabilidad de la frecuencia cardiaca. Sedentarismo. Actividad física. Altura.

\section{Abstract}

Background: Physical exercise and aerobic resistance training have been associated with increased heart rate variability, while sedentary time has been associated with decreased heart rate variability. Objective: To determine if heart rate variability measured at rest is related to sedentary time and time used in various intensities of physical activity, established

\section{Correspondencia:}

*Jaime T. Rojas

E-mail: jaimerojas@udca.edu.co
Disponible en internet: 10-06-2021 Rev Colomb Cardiol. 2021;28(2):189-196 www.rccardiologia.com 0120-5633 / C 2020 Sociedad Colombiana de Cardiología y Cirugía Cardiovascular. Publicado por Permanyer. Este es un artículo open access bajo la licencia CC BY-NC-ND (http://creativecommons.org/licenses/by-nc-nd/4.0/). 
through an objective method (accelerometry), in a group of healthy adults who live 2,600 meters above sea level. Method: Accelerometer measurements were taken in 99 individuals during one week along with one measurement of heart rate variability at rest. Time was divided into sedentary time and time spent in light, moderate and vigorous physical activity. Time (NN interval, SDNN, RMSSD) and frequency (LF, HF, LF/HF) domain parameters were used to analyze heart rate variability. Using regression models, an association was sought between the physical activity and heart rate variability variables. Results: There was a negative association between NN intervals, SDNN and sedentary time, as well as positive associations between the NN interval and light, moderate and vigorous physical activity, and between vigorous physical activity and LF and HF power. All the foregoing associations were significant $(p<0.05)$. Conclusions: In young adults living 2,600 meters above sea level, sedentary time reduces heart rate variability, while vigorous physical activity increases this variability.

Key words: Heart rate variability. Sedentary behavior. Physical activity. Altitude.

\section{Introducción}

La variabilidad de la frecuencia cardiaca puede definirse como la oscilación en el intervalo entre latidos cardiacos consecutivos ${ }^{1}$. El ejercicio físico ${ }^{2}$ y el entrenamiento de resistencia aeróbica ${ }^{3}$ se han asociado con un aumento de la variabilidad de la frecuencia cardiaca, mientras que el tiempo sedentario ${ }^{4}$ se ha relacionado con una disminución de la variabilidad de la frecuencia cardiaca. Esta última también ha tenido utilidad clínica; por ejemplo, se ha usado como parámetro para evaluar el riesgo de enfermedad coronaria ${ }^{5}$, en tanto que la disminución de la variabilidad de la frecuencia cardiaca ha demostrado ser un fuerte predictor de mortalidad y de complicaciones, especialmente de arritmias cardiacas, en pacientes que han sufrido un infarto agudo de miocardio 1 , el cual hace parte de las enfermedades cardiovasculares que son causa importante de mortalidad en el mundo, ya que, de acuerdo con la Organización Mundial de la Salud (OMS), generan 17.9 millones de muertes al año, lo que representa el $31 \%$ de la mortalidad mundial ${ }^{6}$.

La evidencia ha mostrado una relación significativa entre un mayor tiempo en comportamiento sedentario y una mayor mortalidad por enfermedades cardiovasculares (cardiopatía coronaria, accidentes cerebrovasculares, hipertensión arterial), adicional a un mayor riesgo de desarrollar enfermedades cardiovasculares y diabetes mellitus tipo $2^{7}$. Por otra parte, la realización de actividad física en toda la población cuenta con muy buen nivel de evidencia científica, con lo cual se logra demostrar su importancia en la reducción del riesgo de enfermedades cardiovasculares, diabetes mellitus tipo 2 y algunos cánceres. Recientemente, la OMS, el American College of Sports Medicine y la American Heart Association establecieron recomendaciones para toda la población mundial, en las que se asume la actividad física como herramienta para prevenir y tratar enfermedades crónicas no transmisibles 8,9 .
El presente estudio se centró en población universitaria, en su mayoría conformada por adultos entre 18 y 64 años, quienes, con base en las recomendaciones, deberían realizar semanalmente al menos 150 minutos de actividad física aeróbica de intensidad moderada, o disminuir el tiempo pero aumentando la intensidad (la recomendación indica 75 minutos de actividad física aeróbica de intensidad vigorosa) ${ }^{8-10}$.

Para evaluar el tiempo sedentario y el tiempo de actividad física se puede recurrir a diferentes estrategias, tales como cuestionarios, diarios, observación directa o uso de dispositivos, entre los cuales están los acelerómetros ${ }^{11}$. Se ha descrito que el método utilizado con mayor frecuencia para evaluar la actividad física son los cuestionarios ${ }^{12}$, los cuales tienen potenciales ventajas y desventajas ${ }^{11}$; sin embargo, en esta investigación se ha escogido realizar una determinación objetiva de la actividad física mediante acelerometría, ya que es un método no invasivo que tiene entre sus ventajas la habilidad de cuantificar el tiempo gastado en actividades de diferente intensidad ${ }^{13}$. Para determinar la intensidad de la actividad física se utilizan las unidades o equivalentes metabólicos (MET); así, se considera actividad física leve < 3 MET, moderada 3-6 MET y vigorosa > $6 \mathrm{MET}^{14}$.

El objetivo de este estudio fue determinar si la variabilidad de la frecuencia cardiaca medida durante el reposo se relaciona con el tiempo sedentario (entendido como el tiempo que un individuo permanece sentado) y el tiempo utilizado en diferentes intensidades de actividad física (leve, moderada y vigorosa) definidas con un método objetivo (acelerometría) en un grupo de adultos sanos que residen a una altura de 2600 metros sobre el nivel del mar, donde la exposición a la hipoxia puede influir sobre la variabilidad de la frecuencia cardiaca $^{15}$. Estos resultados pueden proporcionar nueva información acerca de la influencia del tiempo sedentario y la actividad física de diferente intensidad sobre 
la modulación autonómica del corazón en sujetos que residen en una altitud moderada sobre el nivel del mar.

\section{Método}

Se realizó un estudio de corte transversal con un muestreo a conveniencia, en el que participaron 116 individuos, todos ellos vinculados como estudiantes, docentes o trabajadores universitarios. Finalmente, se excluyeron 17 que tenían datos incompletos en cuanto a acelerometría o variabilidad de la frecuencia cardiaca, y se incluyeron 99 participantes. A todos se les entregó información relacionada con los objetivos y la metodología del estudio, y se les resolvieron sus dudas antes de la firma del consentimiento informado. Se respetaron los principios éticos de la Declaración de Helsinki para la investigación médica en seres humanos, al igual que las normas establecidas en la Resolución 8430 de 1993 del Ministerio de Salud de la República de Colombia. El estudio contó con la aprobación del Comité de Investigación de la Universidad de Ciencias Aplicadas y Ambientales.

Los participantes asistieron al Laboratorio de Fisiología Experimental de esta misma universidad en dos ocasiones. En la primera visita se realizaron una entrevista médica, una exploración física y un electrocardiograma en reposo, para el cual se utilizó un electrocardiógrafo Schiller Cardiovit MS2010 (Schiller, Switzerland). Como criterios de exclusión se consideraron los antecedentes de enfermedades cardiovasculares, respiratorias, metabólicas o neuromusculares, la presencia de arritmias en el registro electrocardiográfico y las infecciones respiratorias en curso. Al final de la primera visita, cada participante recibía las instrucciones para el uso del acelerómetro y este era activado. Se utilizó un acelerómetro triaxial Actigraph GT3X (Actigraph Corp., Pensacola, FI, USA), con una frecuencia de muestreo de $60 \mathrm{~Hz}$, ajustado con una banda elástica en la cadera a nivel de la línea axilar anterior del lado no dominante, para seguir la misma ubicación utilizada en el estudio de validación de este acelerómetro ${ }^{16}$. Se entregó a cada participante un formato en el cual debía anotar las horas del día en que se retiraba el acelerómetro, la actividad física realizada cada día y las horas a las que se acostaba y se levantaba; lo anterior tenía el propósito de comparar los datos del acelerómetro con los registrados por el participante en dicho formato.

En la segunda visita al laboratorio, la cual se realizaba 7 días después de haber iniciado el registro, se retiraba el acelerómetro y se descargaban los datos de los 7 días de registro al programa Actilife versión 6.13.3. Previo al análisis de los datos se utilizó el parámetro de $\mathrm{Choi}^{17}$ para validar el tiempo de uso del acelerómetro. Los momentos determinados como tiempo válido de uso del acelerómetro a través de este parámetro se compararon con los reportados por cada participante en el formato de uso del acelerómetro, buscando su coincidencia. Se utilizó el algoritmo de Freedson ${ }^{18}$ para determinar el tiempo sedentario y el nivel de actividad física.

En la segunda visita también se realizó la medición de la variabilidad de la frecuencia cardiaca. Desde la primera visita al laboratorio, los participantes recibían instrucciones para que siguieran las siguientes recomendaciones antes de la segunda medición: dormir al menos 8 horas la noche previa a la medición, no realizar ejercicio físico vigoroso, evitar la ingesta de café, chocolate, alcohol y bebidas energizantes 24 horas antes, y no haber consumido medicamentos que modifiquen la actividad autonómica. La medición de la variabilidad de la frecuencia cardiaca se realizó con un monitor de frecuencia cardiaca Polar RS800CX (Polar Electro, Kempele, Finland), con los participantes ubicados en decúbito supino en una camilla y con un reposo previo de 15 minutos. Durante la medición, los participantes permanecían en silencio y con los ojos cerrados, y se evitaba la generación de cualquier estímulo auditivo con el propósito de mantener un ambiente tranquilo. Las mediciones se realizaron entre las 10:00 y las 14:00 horas. La temperatura ambiental en el laboratorio de fisiología experimental fue de $20^{\circ} \mathrm{C}$ y la humedad relativa del ambiente permaneció en el $50 \%$. La duración de cada medición fue de 30 minutos, después de los cuales se retiraba el monitor de frecuencia cardiaca y los datos eran descargados al programa Polar ProTrainer 5, para luego ser exportados a la versión libre del programa Kubios HRV versión 2.2, en el cual se realizó el análisis de la variabilidad de la frecuencia cardiaca utilizando un nivel medio de corrección de artefactos. La variabilidad de la frecuencia cardiaca se evaluó utilizando métodos del dominio del tiempo y del dominio de la frecuencia descritos en la Guía de estándares de medida, interpretación fisiológica y uso clínico de la variabilidad de la frecuencia cardiaca ${ }^{1}$. Se utilizaron tres parámetros del dominio del tiempo: el intervalo normal (NN), que es el intervalo entre complejos QRS adyacentes que resulta de la despolarización del nodo sinusal (puede ser interpretado también como el intervalo RR), la desviación estándar de los intervalos NN (SDNN) y la raíz cuadrada del promedio de las diferencias al cuadrado entre 
intervalos normales adyacentes (RMSSD). Se utilizaron también tres parámetros del dominio de la frecuencia obtenidos a partir de la transformada rápida de Fourier: la potencia de la baja frecuencia (LF) en el rango de 0,04 a $0,15 \mathrm{~Hz}$, la potencia de la alta frecuencia (HF) en el rango de 0,15 a $0,4 \mathrm{~Hz}$ y la relación entre estas dos (LF/HF).

El análisis estadístico se realizó con el programa SAS versión 9.0. Se agruparon los datos en variables relacionadas con la actividad física y el tiempo sedentario (variables independientes) y variables relacionadas con la variabilidad de la frecuencia cardiaca (variables dependientes). Se realizó un análisis estadístico descriptivo para obtener los valores medios y la desviación estándar de cada variable, y posteriormente se realizaron análisis de regresión lineal, cuadrática y cúbica. Se usaron transformaciones logarítmicas para comparar las variables del dominio de la frecuencia con el tiempo sedentario y la actividad física. Para los modelos considerados en el estudio se tuvo en cuenta un nivel de significancia de $p<0.05$.

\section{Resultados}

Completaron todas las mediciones 99 participantes, de los cuales 59 (59.6\%) eran mujeres y 40 (40.4\%) eran hombres. El $16.2 \%$ de los participantes reportaron ser fumadores. Las mediciones se realizaron en tres grupos diferentes: 73 estudiantes (63\% mujeres), 18 trabajadores (55.6\% mujeres) y 8 docentes $(37.5 \%$ mujeres). La edad media fue de 24.9 años y el índice de masa corporal medio fue de $23.1 \mathrm{~kg} / \mathrm{m}^{2}$; presentaron sobrepeso 23 participantes y un participante presentó bajo peso. Los demás datos generales de la población se muestran en la tabla 1.

Dentro de la medición objetiva del tiempo sedentario y la actividad física se encontró que los participantes permanecieron en promedio un $82.33 \%$ del tiempo en actividades sedentarias, un $13.54 \%$ del tiempo en actividad física de intensidad leve, un $3.98 \%$ del tiempo en actividad moderada y un $0.12 \%$ del tiempo en actividad física vigorosa. Todos los participantes cumplieron con la recomendación de la OMS de realizar al menos 150 minutos a la semana de actividad física moderada o vigorosa.

La otra variable del dominio del tiempo en la que se encontró una asociación significativa a través de un modelo de regresión cúbico fue la SDNN, la cual presentó una asociación negativa con el tiempo total de episodios sedentarios ( $B=-0.0001 ; p=0.04$ ). Los diferentes niveles de actividad física no se asociaron con la SDNN. En cuanto a la RMSSD, no se hallaron asociaciones significativas entre esta y las diferentes variables de sedentarismo y actividad física.

En lo que respecta a los indicadores de variabilidad de la frecuencia cardiaca en el dominio de la frecuencia, se halló una asociación positiva entre la actividad física vigorosa y la potencia de la baja frecuencia $(B=0.0092 ; p=0.03)$, y entre la actividad física vigorosa y la potencia de la alta frecuencia $(B=0.01$; $p=0.03)$. No se observó una asociación significativa entre el tiempo sedentario y la potencia de LF y HF, al igual que tampoco la hubo para los otros niveles de actividad física (ligera y moderada). La tabla 2 muestra las asociaciones encontradas y se destacan aquellas que fueron significativas.

\section{Discusión}

En este estudio se encontró que la actividad física de intensidad vigorosa se asoció de manera positiva con la variabilidad de la frecuencia cardiaca determinada durante el reposo en estudiantes, docentes y trabajadores universitarios, mientras que el tiempo sedentario se asoció de manera negativa; lo anterior indica que el ejercicio físico vigoroso mejora la modulación autonómica del corazón durante el reposo, mientras que la sedestación prolongada la altera. Estos hallazgos guardan relación con estudios realizados en individuos sedentarios que hicieron entrenamiento a intervalos de alta intensidad por un periodo de 8 semanas y presentaron modificaciones significativas positivas en la variabilidad de la frecuencia cardiaca y, por tanto, en la modulación autonómica del corazón ${ }^{19}$.

Como hallazgo adicional, se encontró que la edad se asocia de manera negativa con los índices del dominio del tiempo (RMSSD) y de la frecuencia (HF) de la variabilidad de la frecuencia cardiaca. La asociación hallada en el presente estudio entre la edad y la variabilidad de la frecuencia cardiaca guarda relación con lo descrito en otros estudios, en los que se ha encontrado que la edad se asocia con un aumento de la actividad simpática y una reducción de la actividad parasimpática ${ }^{20}$. Como causas de este cambio de la variabilidad de la frecuencia cardiaca asociado a la edad se han propuesto alteraciones en los mecanismos de señalización relacionados con los receptores del sistema nervioso autónomo y mecanismos intrínsecos de las células marcapasos del corazón ${ }^{21}$.

En este estudio, el 100\% de los participantes cumplieron con las recomendaciones de actividad física de 
Tabla 1. Variables generales de la población, medidas objetivas de los tiempos de sedentarismo y actividad física, y parámetros de variabilidad de la frecuencia cardiaca

\begin{tabular}{|c|c|c|}
\hline Variable & Media & Desviación estándar \\
\hline Edad (años) & 24.9 & 9.5 \\
\hline Presión arterial media $(\mathrm{mmHg})$ & 86.4 & 7.1 \\
\hline Índice de masa corporal ( $\left.\mathrm{kg} / \mathrm{m}^{2}\right)$ & 23.1 & 3.4 \\
\hline Tiempo sedentario (minutos/semana) & 7738.3 & 848.5 \\
\hline Tiempo en actividad física leve (minutos/semana) & 1269.8 & 337 \\
\hline Tiempo en actividad física moderada (minutos/semana) & 372.5 & 135.4 \\
\hline Tiempo en actividad física vigorosa (minutos/semana) & 10.9 & 19.4 \\
\hline Frecuencia cardiaca (latidos/minuto) & 71.4 & 9.2 \\
\hline Intervalo NN (ms) & 861.2 & 111.8 \\
\hline SDNN (ms) & 77 & 27.9 \\
\hline RMSSD (ms) & 55.7 & 28.6 \\
\hline $\operatorname{LF}\left(\mathrm{ms}^{2}\right)$ & 1460.3 & 1065.4 \\
\hline $\mathrm{HF}\left(\mathrm{ms}^{2}\right)$ & 1362.2 & 1382.2 \\
\hline LF/HF & 2.4 & 4.7 \\
\hline
\end{tabular}

LF: potencia de la baja frecuencia; HF: potencia de la alta frecuencia; RMSSD: raíz cuadrada del promedio de las diferencias al cuadrado entre intervalos normales adyacentes; SDNN: desviación estándar de los intervalos normales.

En los modelos de regresión, la edad se asoció de manera negativa con la RMSSD ( $B=-1.032 ; p=0.0051)$ y con la HF ( $B=-43.2 ; p=0.0292)$, y el el intervalo NN fue el índice de variabilidad de la frecuencia cardiaca que se relacionó con todas las variables independientes (tiempo sedentario y tiempos de actividad física ligera, moderada y vigorosa).

la $\mathrm{OMS}^{8}$. Por otro lado, la Encuesta Nacional de la Situación Nutricional (ENSIN) de 2015 reportó que un $42.7 \%$ de las mujeres y un $61.1 \%$ de los hombres colombianos de 18 a 64 años cumplían con estas recomendaciones ${ }^{22}$. Estas diferencias en los porcentajes de cumplimiento entre la ENSIN 2015 y el presente estudio pueden deberse a que en este último se recurrió a un muestreo no probabilístico y, por ende, el poder de inferencia es menor que el de la ENSIN 2015.

Los hallazgos del presente estudio guardan relación con uno realizado en adultos jóvenes, en el que se encontró que las horas de actividad física vigorosa, pero no las de actividad física moderada, fueron predictores de mayores índices en el dominio del tiempo y de la frecuencia de la variabilidad de la frecuencia cardiaca $^{2}$, y con otro estudio en sujetos de 45 a 68 años de edad en el que se halló que la actividad física vigorosa se asoció con una mayor variabilidad de la frecuencia cardiaca ${ }^{23}$. De igual forma, se ha encontrado que en hombres adultos sedentarios un programa de ejercicio de intensidad moderada a vigorosa incrementa las medidas de los dominios del tiempo y la frecuencia de la variabilidad de la frecuencia cardiaca $^{24}$.

En relación con el cambio de la frecuencia cardiaca que ocurre durante el ejercicio, se ha observado que se mantiene activa la modulación parasimpática sobre el corazón, pero se presenta una reducción progresiva de su actividad hasta la máxima intensidad del ejercicio, mientras que acontece una situación opuesta con la actividad simpática; es decir, un aumento de esta hasta la máxima intensidad del ejercicio ${ }^{25}$. Sin embargo, si bien los cambios descritos serían aquellos que se presentan durante el ejercicio, a partir de los hallazgos de este estudio se puede concluir que la actividad física vigorosa se asocia con mayor variabilidad de la frecuencia cardiaca durante el reposo, lo cual se puede considerar un factor protector, ya que se han encontrado asociaciones entre una menor variabilidad de la frecuencia cardiaca y una mayor mortalidad ${ }^{26}$; además, la reducción del comportamiento sedentario se ha relacionado con un aumento en la expectativa de vida ${ }^{27}$.

En un reciente estudio se encontró que la actividad física muy vigorosa (> $8 \mathrm{MET}$ ) tiene una correlación 
Tabla 2. Modelos de regresión para asociar el tiempo sedentario y los diferentes niveles de actividad física con los parámetros de variabilidad de la frecuencia cardiaca en el dominio del tiempo y de la frecuencia

\begin{tabular}{|c|c|c|c|}
\hline Parámetros & Tiempo sedentario o nivel de actividad física & B & $p<0.05$ \\
\hline \multirow[t]{4}{*}{ Intervalos NN } & Tiempo sedentario & -0.0004 & $0.01^{*}$ \\
\hline & Actividad física ligera & 0.0680 & 0.04 \\
\hline & Actividad física moderada & 0.1960 & 0.02 \\
\hline & Actividad física vigorosa & 1.2400 & 0.03 \\
\hline \multirow[t]{4}{*}{ SDNN } & Tiempo sedentario & -0.0001 & $0.04^{*}$ \\
\hline & Actividad física ligera & -0.0094 & 0.26 \\
\hline & Actividad física moderada & -0.0045 & 0.83 \\
\hline & Actividad física vigorosa & -13.7360 & 0.15 \\
\hline \multirow[t]{4}{*}{ RMSSD } & Tiempo sedentario & 0.0065 & 0.07 \\
\hline & Actividad física ligera & -0.0152 & 0.08 \\
\hline & Actividad física moderada & -0.0064 & 0.77 \\
\hline & Actividad física vigorosa & -0.9322 & 0.34 \\
\hline \multirow[t]{4}{*}{ InLF } & Tiempo sedentario & 0.0001 & 0.19 \\
\hline & Actividad física ligera & -0.0003 & 0.16 \\
\hline & Actividad física moderada & 0.0006 & 0.35 \\
\hline & Actividad física vigorosa & 0.0092 & 0.03 \\
\hline \multirow[t]{4}{*}{ InHF } & Tiempo sedentario & 0.0002 & 0.07 \\
\hline & Actividad física ligera & -0.0005 & 0.13 \\
\hline & Actividad física moderada & -0.0002 & 0.81 \\
\hline & Actividad física vigorosa & 0.0100 & 0.03 \\
\hline \multirow[t]{4}{*}{ InLF/HF } & Tiempo sedentario & -0.0002 & 0.24 \\
\hline & Actividad física ligera & 0.0000 & 0.92 \\
\hline & Actividad física moderada & 0.0003 & 0.74 \\
\hline & Actividad física vigorosa & -0.0064 & 0.35 \\
\hline
\end{tabular}

LF: potencia de la baja frecuencia; HF: potencia de la alta frecuencia; NN: intervalos normales; RMSSD: raíz cuadrada del promedio de las diferencias al cuadrado entre intervalos normales adyacentes; SDNN: desviación estándar de los intervalos normales.

*Corresponden a un modelo cúbico de regresión. No se presentaron asociaciones significativas entre la otra variable analizada en el dominio del tiempo que correspondió a la RMSSD y las diferentes variables independientes, ni entre la relación entre la potencia de la baja frecuencia y la potencia de la alta frecuencia (LF/HF) con las variables independientes.

positiva con la variabilidad de la frecuencia cardiaca; sin embargo, los investigadores de dicho estudio sugieren que no hay un beneficio adicional de la actividad física muy vigorosa sobre la variabilidad de la frecuencia cardiaca con tiempos mayores de 21 minutos a la semana, a esta intensidad de actividad física ${ }^{28}$. De acuerdo con lo anterior, se sugiere que aumentar la intensidad del ejercicio físico vigoroso a muy vigoroso podría no tener efectos adicionales sobre la variabilidad de la frecuencia cardiaca.
Debido a la fuerte relación entre la acelerometría, el gasto energético humano y el movimiento, se han utilizado acelerómetros en diferentes tipos de estudios para evaluar la actividad física y el comportamiento sedentario ${ }^{29}$. En un estudio en el que se evaluó el tiempo sedentario con estos instrumentos, se describió que el tiempo de sedestación ocupacional tiene asociaciones negativas significativas con los índices de tiempo y frecuencia de la variabilidad de la frecuencia cardiaca $^{4}$, hallazgo que concuerda con lo encontrado 
en el presente estudio, en el que el tiempo sedentario se asoció con una menor duración del intervalo entre latidos cardiacos en reposo, lo que a la vez indica una mayor frecuencia cardiaca y un predominio de la actividad simpática sobre la parasimpática. No obstante, a diferencia de otros trabajos ${ }^{4,30}$, en el presente estudio no se hallaron asociaciones significativas entre el tiempo sedentario y los índices del dominio de la frecuencia de la variabilidad de la frecuencia cardiaca.

Si bien las asociaciones encontradas en este estudio entre la actividad física vigorosa, el tiempo sedentario y la variabilidad de la frecuencia cardiaca se han descrito con anterioridad, se requerirían estudios adicionales para evaluar si la aclimatación a la altura puede ser un factor que influye para modificar las otras asociaciones de la actividad física de menor intensidad (leve y moderada) con la variabilidad de la frecuencia cardiaca, ya que se ha descrito que la exposición a la altura produce cambios en el sistema cardiovascular ${ }^{31}$. Consideramos que la variabilidad de la frecuencia cardiaca puede ser útil como marcador temprano de riesgo cardiovascular, como ya han propuesto otros autores ${ }^{30}$.

\section{Conclusiones}

El tiempo sedentario se asoció con una menor variabilidad de la frecuencia cardiaca determinada a partir del análisis de las variables en el dominio del tiempo (intervalos NN y SDNN), mientras que la actividad física vigorosa se relacionó con una mayor variabilidad de la frecuencia cardiaca determinada a partir del análisis en los dominios del tiempo (intervalos NN) y de la frecuencia (HF).

En adultos jóvenes sanos, la actividad física vigorosa se asocia con una mejora de la modulación autonómica del corazón durante el reposo.

\section{Limitaciones}

Este estudio tiene algunas limitaciones metodológicas. En este estudio no se evaluó el estrés en los participantes, ya que factores emocionales pueden influir en el balance autonómico ${ }^{32}$ y, por lo tanto, en la variabilidad de la frecuencia cardiaca, no es posible concluir si el estrés o la ansiedad tuvieron alguna influencia en los índices de variabilidad de la frecuencia cardiaca. Tampoco se realizó una división entre el tiempo sedentario ocupacional y el tiempo sedentario durante el descanso, los cuales podrían tener una influencia diferente sobre la variabilidad de la frecuencia cardiaca, como se ha descrito en otros estudios ${ }^{4,33}$.

\section{Agradecimientos}

Los autores agradecen a los estudiantes, docentes y trabajadores universitarios, quienes con su participación como voluntarios hicieron posible el desarrollo de esta investigación.

\section{Financiamiento}

Esta investigación fue financiada por la Universidad de Ciencias Aplicadas y Ambientales (Bogotá D.C., Colombia).

\section{Conflicto de intereses}

Los autores declaran que no existe conflicto de intereses.

\section{Responsabilidades éticas}

Protección de personas y animales. Los autores declaran que los procedimientos seguidos se conformaron a las normas éticas del comité de experimentación humana responsable y de acuerdo con la Asociación Médica Mundial y la Declaración de Helsinki.

Confidencialidad de los datos. Los autores declaran que han seguido los protocolos de su centro de trabajo sobre la publicación de datos de pacientes.

Derecho a la privacidad y consentimiento informado. Los autores han obtenido el consentimiento informado de los pacientes y/o sujetos referidos en el artículo. Este documento obra en poder del autor de correspondencia.

\section{Bibliografía}

1. Malik M, Bigger J, Camm A, Kleiger R, Malliani A, Moss A, et al. Heart rate variability: standards of measurement, physiological interpretation, and clinical use. Eur Heart J. 1996:17:354-81.

2. May R, McBerty V, Zaky A, Gianotti M. Vigorous physical activity predicts higher heart rate variability among younger adults. J Physiol Anthropol. 2017;36:24. DOI: 10.1186/s40101-017-0140-z

3. Melanson E, Freedson $P$. The effect of endurance training on resting hear rate variability in sedentary adult males. Eur J Appl Physiol. 2001;85:442-9.

4. Hallman D, Sato T, Kristiansen J, Gupta N, Skotte J, Holtermann A. Prolonged sitting is associated with attenuated heart rate variability during sleep in blue-collar workers. Int J Environ Res Public Health. 2015;12:14811-27.

5. Yoo C, Lee K, Yi S, Kim J, Kim H. Association of heart rate variability with the Framingham Risk Score in healthy adults. Korean J Fam Med. 2011;32:334.

6. Cardiovascular diseases. World Health Organization. 2019. [Citado 18 abril 2019]. Disponible en: https://www.who.int/cardiovascular_diseases/en/. 
7. 2018 Physical Activity Guidelines Advisory Committee. 2018 Physical Activity Guidelines Advisory Committee Scientific Report. Washington, DC: U.S. Department of Health and Human Services; 2018.

8. Organización Mundial de la Salud. Recomendaciones mundiales sobre actividad física para la salud. Ginebra: OMS; 2010.

9. Riebe D, Ehrman J, Liguori G, Magal M. ACSM's guidelines for exercise testing and prescription. 10th ed. Philadelphia: Wolters Kluwer; 2017.

10. Haskell WL, Lee IM, Pate RR, Powell KE, Blair SN, Franklin BA, et al Physical activity and public health: updated recommendation for adults from the American College of Sports Medicine and the American Heart Association. Med Sci Sports Exerc. 2007;39:1423-34.

11. Sylvia L, Bernstein E, Hubard J, Keating L, Anderson E. A practica guide to measuring physical activity. J Acad Nutr Diet. 2014;114:199-208.

12. Castillo-Retamal M, Hinckson EA. Measuring physical activity and sedentary behaviour at work: a review. Work. 2011;40:345-57.

13. Hills A, Mokhtar N, Byrne N. Assessment of physical activity and energy expenditure: an overview of objective measures. Front Nutr. 2014;1. Disponible en: https://doi.org/10.3389/fnut.2014.00005

14. Ainsworth B, Haskell W, Whitt M, Irwin M, Swartz A, Strath S, et al. Compendium of physical activities: an update of activity codes and MET intensities. Med Sci Sports Exerc. 2000;32(Suppl):S498-S516.

15. Oliveira A, Rohan $P$, Gonçalves $T$, Soares $P$. Effects of hypoxia on heart rate variability in healthy individuals: a systematic review. Int J Cardiovasc Sci. 2017;30:251-61.

16. Sasaki J, John D, Freedson P. Validation and comparison of ActiGraph activity monitors. J Sci Med Sport. 2011;14:411-6.

17. Choi L, Liu Z, Matthews C, Buchowski M. Validation of accelerometer wear and nonwear time classification algorithm. Med Sci Sports Exerc. 2011;43:357-64.

18. Freedson P, Melanson E, Sirard J. Calibration of the Computer Science and Applications, Inc. accelerometer. Med Sci Sports Exerc. 1998;30:777-81.

19. Alansare A, Alford K, Lee S, Church T, Jung $H$. The effects of high-intensity interval training vs. moderate-intensity continuous training on heart rate variability in physically inactive adults. Int J Environ Res Public Health. 2018;15:1508.

20. Estévez-Báez M, Carricarte-Naranjo C, Jas-García J, Rodríguez-Ríos E, Machado C, Leisman G, et al. Influence of heart rate, age, and gende on heart rate variability in adolescents and young adults. Adv Exp Med Biol. 2019;1133:19-33.
21. Yaniv Y, Ahmet I, Tsutsui K, Behar J, Moen J, Okamoto Y, et al. Deterioration of autonomic neuronal receptor signaling and mechanisms intrinsic to heart pacemaker cells contribute to age-associated alterations in heart rate variability in vivo. Aging Cell. 2016;15:716-24.

22. Encuesta Nacional de la Situación Nutricional 2015. Nota de Política. Ministerio de Salud, Prosperidad Social, Instituto Nacional de Salud, Bienestar Familiar, Universidad Nacional de Colombia. 2015. Disponible en: http://www.prosperidadsocial.gov.co/temporales/Encuesta\%20Nacional\%20de \%20la\%20Situacio\%CC\% 81 n\%20Nutricional $\% 20-\% 20$ ENSIN\%202015.pdf.

23. Rennie K, Hemingway $H$, Kumari M, Brunner E, Malik M, Marmot M. Effects of moderate and vigorous physical activity on heart rate variability in a British Study of Civil Servants. Am J Epidemiol. 2003;158:135-43.

24. Melanson $E$, Freedson $P$. The effect of endurance training on resting heart rate variability in sedentary adult males. Eur J Appl Physiol. 2001;85:442-9.

25. White $D$, Raven $P$. Autonomic neural control of heart rate during dynamic exercise: revisited. J Physiol. 2014;592:2491-500.

26. Tsuji H, Venditti F, Manders E, Evans J, Larson M, Feldman C, et al. Reduced heart rate variability and mortality risk in an elderly cohort. The Framingham Heart Study. Circulation. 1994;90:878-83.

27. Katzmarzyk P, Lee I. Sedentary behaviour and life expectancy in the USA: a cause-deleted life table analysis. BMJ Open. 2012;2:e000828.

28. de Sousa T, Ostoli T, Sperandio E, Arantes R, Gagliardi A, Romiti M, et al. Dose-response relationship between very vigorous physical activity and cardiovascular health assessed by heart rate variability in adults: cross-sectional results from the EPIMOV study. PLoS One. 2019;14:e0210216.

29. Luiselli J, Fischer A. Computer-assisted and web-based innovations in psychology, special education, and health. Philadelphia: Elsevier; 2016. Disponible en: https://doi.org/10.1016/C2014-0-01763-7

30. Zaffalon Júnior J, Viana A, de Melo G, De Angelis K. The impact of sedentarism on heart rate variability (HRV) at rest and in response to mental stress in young women. Physiol Rep. 2018;6:e13873.

31. Naeije R. Physiological adaptation of the cardiovascular system to high altitude. Prog Cardiovasc Dis. 2010:52:456-66.

32. Friedman B, Thayer J. Autonomic balance revisited: panic anxiety and heart rate variability. J Psychosom Res. 1998;44:133-51.

33. Sato T, Hallman D, Kristiansen J, Skotte J, Holtermann A. Different autonomic responses to occupational and leisure time physical activities among blue-collar workers. Int Arch Occup Environ Health. 2017;91:293-304. 